\title{
PREDICTORS OF IN-HOSPITAL MORTALITY IN A MULTI-ETHNIC SOUTHEAST ASIAN SURGICAL INTENSIVE CARE UNIT
}

Keith J. Yong1', Joseph W. E. Wong ${ }^{1}$, Ming Ann Sim², Chai Rick Soh ${ }^{3}$, Lian Kah Ti², Sophia T. H. Chew ${ }^{4,5}$

${ }^{1}$ Yong Loo Lin School of Medicine, National University of Singapore, Singapore, ${ }^{2}$ Department of Anaesthesia, National University Health System, Singapore, ${ }^{3}$ Department of Anaesthesia and Surgical Intensive Care, Singapore General Hospital, Singapore, ${ }^{4}$ Department of Anaesthesiology, Singapore General Hospital, Singapore, ${ }^{5}$ Duke-National University of Singapore Graduate Medical School, Singapore.

\section{Introduction}

Despite rapid advancements in intensive care management, the mortality of patients admitted to the Intensive Care Unit (ICU) ranges from $7 \%$ to $40 \% .^{1,2}$ Previous studies in Middle Eastern and South American populations have identified several predictors of surgical ICU (SICU) mortality, including emergency surgery, high Acute Physiology and Chronic Heath Evaluation II (APACHE) score, low Glasgow Coma Scale (GCS), use of mechanical ventilation, and haemodynamic and metabolic instability. ${ }^{2,3}$

Given our multi-ethnic population with potentially different risk factors, we aimed to identify similarities between predictors of SICU mortality in our local population and other previously researched populations.

\section{Methods}

Following IRB approval, we conducted a retrospective cohort study of 858 post-operative patients admitted to a single-center SICU of a tertiary hospital from February 2015 to October 2016. APACHE scores were calculated on the first day of admission. The primary outcome studied was in-hospital mortality, defined as death occurring during the hospital stay. The length of SICU stay was evaluated as a secondary outcome.

\section{Results}

The in-hospital mortality rate was $20.6 \%$. The mean age of patients was 61.2 , and $54.7 \%$ were male.

Patients who were more likely to die were older, Malay, underwent emergency surgery, had higher APACHE scores, lower GCS, anemia, haemodynamic and metabolic instability, and acute kidney injury (table 1). Multivariate analysis showed age, APACHE score, hypernatremia, hypokalemia, hypotension, acidosis and elevated creatinine to be independent predictors of mortality (table 2).

Patients who died also stayed in the SICU for an average of 3 days longer, utilizing more ICU resources.

Table 1: Predictors of SICU mortality (univariate analysis)

\begin{tabular}{|l|l|l|l|}
\hline \multirow{2}{*}{ Variable } & \multicolumn{2}{|c|}{ N (\%) / Mean \pm SD } & p- \\
\cline { 2 - 4 } & No Mortality & Mortality & value \\
\hline Age (Years) & $59.5 \pm 15.8$ & $67.7 \pm 14.4$ & $<0.001$ \\
\hline Ethnicity (Malay) & $58(8.5 \%)$ & $18(10.2 \%)$ & 0.043 \\
\hline Type of Surgery (Emergency) & $189(27.8 \%)$ & $89(50.3 \%)$ & $<0.001$ \\
\hline APACHE Score & $14.19 \pm 7.34$ & $24.20 \pm 8.48$ & $<0.001$ \\
\hline Low Haematocrit (<36\%) & $467(69 \%)$ & $154(87 \%)$ & $<0.001$ \\
\hline Hypernatremia (>145mmol/L) & $41(6.1 \%)$ & $35(19.8 \%)$ & $<0.001$ \\
\hline Hypokalemia (<3.5mmol/L) & $82(12.1 \%)$ & $49(27.7 \%)$ & $<0.001$ \\
\hline Acidosis (pH < 7.35) & $288(43.3 \%)$ & $89(51.4 \%)$ & 0.055 \\
\hline Hypotension (MAP < 70) & $100(14.7 \%)$ & $71(40.1 \%)$ & $<0.001$ \\
\hline Acute Kidney Injury (AKIN 1) & $100(15.4 \%)$ & $35(24.5 \%)$ & 0.009
\end{tabular}

Table 2: Predictors of SICU mortality (multivariate analysis)

\begin{tabular}{|l|l|l|l|}
\hline Variable & Odds Ratio & $\begin{array}{l}\text { 95\% Confidence } \\
\text { Interval }\end{array}$ & -value \\
\hline Age & 1.024 & $1.006-1.042$ & 0.010 \\
\hline APACHE score & 1.076 & $1.038-1.114$ & $<0.001$ \\
\hline Hypernatremia & 2.064 & $1.066-3.996$ & 0.032 \\
\hline Hypokalemia & 1.713 & $1.002-2.927$ & 0.049 \\
\hline Hypotension & 1.699 & $1.029-2.804$ & 0.038 \\
\hline Acidosis & 1.567 & $1.005-2.442$ & 0.047 \\
\hline $\begin{array}{l}\text { Highest Creatinine in } \\
\text { 1st 24 hours }\end{array}$ & 1.003 & $1.001-1.004$ & $<0.001$ \\
\hline
\end{tabular}

Figure 1: Incidence of Predictors in SICU Population

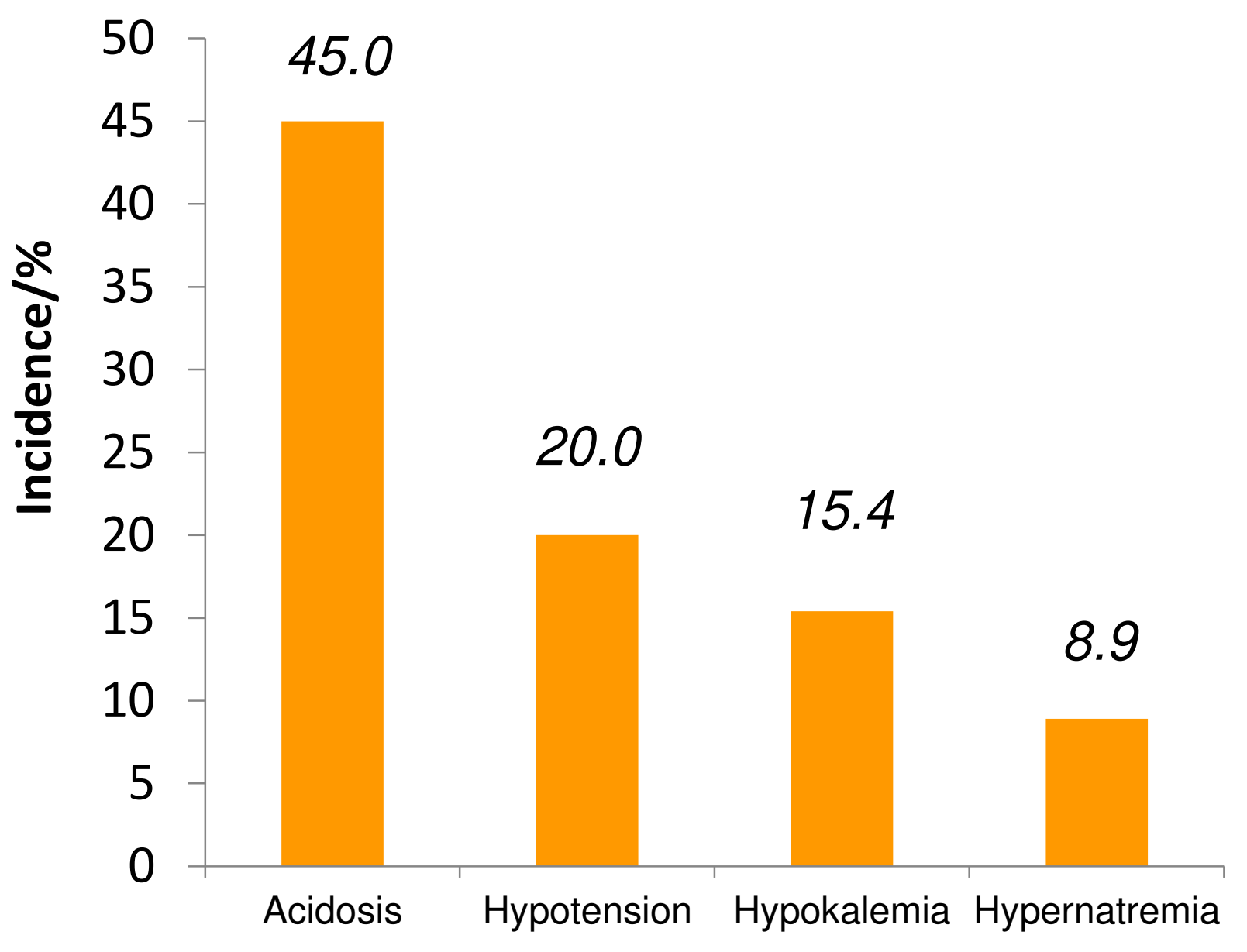

\section{Conclusion}

Hypernatremia is associated with the highest risk of mortality while acidosis is the most common predictor (figure 1). This is likely explained by critically ill surgical patients developing acidosis secondary to organ failure and requiring large volumes of bicarbonate infusion. The effect of hypernatremia on mortality is also consistent with other studies. ${ }^{2}$ Hypernatremia in the ICU is likely to be iatrogenic in nature, and thus, largely preventable.

Strategies targeting modifiable risk factors such as the early treatment of metabolic derangements should be evaluated and may improve patient outcomes.

\section{References}

1. Siddiqui S. Indian J Crit Care Med. 2015

2. Alansari MA et al. Saudi J Anaesth. 2016

3. Uzman S et al. Hippokratia 2016 